\title{
Molecular detection of Virulence Factors genes Associated with Immune Resistance in Klebsiella pneumonia
}

\author{
Nihad Jawad Kadhim ${ }^{1}$, Hayder Shkhair Al-Janabi ${ }^{2}$, Mohanad Jawad Kadhim ${ }^{3}$ \\ ${ }^{1}$ M.Sc., ${ }^{2}$ Prof. Dr., ${ }^{3}$ Assist.Prof. Dr., Department of Biotechnology, College of Biotechnology, Al-Qasim Green \\ University, Babylon, Iraq
}

\begin{abstract}
The current study was aimed to genotypically detection of genes encode outer membrane proteins related with human serum resistance. A total of 33 isolates were identified as Klebsiella pneumonia according to the manual cultural characteristics and biochemical tests and then confirmed by Vitek -2 system. The number of isolates according to site of infection were $14(42.4 \%)$ isolates from wound infection, $15(45.4 \%)$ isolates from urine, 1(3.03\%) isolates from blood, 1(3.03\%) isolates from vagina, 1(3.03\%) from ear swab and finally $1(3.03 \%)$ isolates from stool. The present study demonstrated a positive relationship between the ability of bacterial isolates to antibiotics resistance and the occurrence of some virulence genes. The molecular detection of some virulence genes ( $h t r A, \operatorname{rmp} A, m r k A$ and iss) was done by conventional PCR technique. It was found that ,high occurrence of htrA gene was observed among Klebsiella pneumonia isolates which was found in $26(78 \%$ ) of isolates at $1071 \mathrm{bp}$, then $17(51 \%)$ isolate were positive for $\mathrm{rmpA}$ gene at 967 bp,whereas $m r k A$ gene was recorded in $5(15.15 \%)$ isolates at $862 \mathrm{bp}$, and only $4(12.12 \%)$ isolates gave positive result for $i s s$ gene at $450 \mathrm{bp}$.
\end{abstract}

Key words: Klebsiella pneumonia, molecular detection, virulence gene.

\section{Introduction}

Klebsiella pneumonia is commonly dispersed in the gastrointestinal, urinary, and respiratory tracts of healthy people ${ }^{1}$. It causes opportunistic infections and nosocomial infections; it is a popular hospitalacquired pathogen causing severe respiratory infections such as pneumonia. Other infections caused by this organism include urinary tract infection, wound infection, abscesses, sepsis, inflammation, and diarrhea ${ }^{1}$. K. pneumonia have different virulence factors which provide bacteria the capacity to invade the host, such as capsular polysaccharide, lipopolysaccharide, serum resistance, siderophores production, fimbriae and other factors such as the production of urease and enterotoxin 2,3. However, antibiotic resistance properties are the major factor in its pathogenicity that it resists for a wide spectrum of antibiotics and specially $\beta$-lactam antibiotics ,particularly those involved in nosocomial diseases ${ }^{4,5}$. The $m r k A$ gene encodes the fimbrial subunit, which is polymerized to form the helical fimbrial shaft the adhesive subunit, with the ability to bind to collagen molecules, is encoded by $m r k \mathrm{D}$ and located at the tip of the fimbriae ${ }^{6}$. Iss gene has been localized to gain virulence plasmids and shares sturdy similarities with the bor gene from bacteriophage ${ }^{7}$. htr A gene is now of interest because htrA mutant strains of several gramnegative bacteria have been shown to be attenuated in animal models and can also be used as vaccines ${ }^{7}$. rmpA gene the regulator of the mucoid phenotype $\mathrm{A}(\mathrm{rmpA})$ is a mucoid factor which is responsible for the regulation of the capsular polysaccharide biosynthesis Cheng (2010). Wide spreading of K. pneumonia infections and antibiotic resistance properties for a large spectrum of antibiotics appeared among K. pneumonia isolates were a cause to study the prevalence of some virulence genes associated with serum resistance among K. pneumonia isolates in Babylon province, Iraq as indicator for distribution of the bacterial infections.

\section{Materials and Method}

\section{Isolation and Identification}

Thirty-three isolates of Klebsiella pneumonia were 
collected from different clinical sources (urine, blood, stool, vagina, ear swab and wound) from patients were admitted to Hilla teaching hospital, AL-Hashimia general Hospital and medical city of Merjan in Babylon province during the period from July 2019 to December 2020. Each specimen was inoculated on selective media and identified by biochemical reaction according to the diagnostic procedures recommended in (Forbes et al., 2007). Diagnosis was established by the automated method Vitek ${ }^{\circledR} 2$ (BioMérieux).

\section{Molecular Detection of Some Virulence Factors}

We using PCR technique for detection of some virulence gene include (htrA, rmpA, mrkA and iss)

\section{Extraction of Bacterial DNA}

The whole bacterial DNA was extracted from bacterial isolates by using Genomic DNA Mini Bacteria Kit that provided by the company favrgen, tiwan). The DNA solution was stored at $-20^{\circ} \mathrm{C}$ till used in PCR.

\section{PCR Amplifications}

Recognition of virulence genes in Klebsiella pneumonia isolates was achieved by amplifying PCR. The PCR primers were provided by (Techne (UK). The product size and sequences of the PCR primers are showed in Table 1.

Table 1: The size and sequences of primers used in current study

\begin{tabular}{|c|c|c|c|c|}
\hline Gene name & \multicolumn{2}{|c|}{ Primer sequence (5'-3') } & Product size (bp) & References \\
\hline \multirow{2}{*}{$\mathrm{rmpA}$} & $\mathrm{F}$ & CGCAGAAGTATTCCGTTGGT & \multirow{2}{*}{967} & \multirow{2}{*}{$\begin{array}{l}\text { AB289644.1 } \\
\text { This study }\end{array}$} \\
\hline & $\mathrm{R}$ & TTTGTTAGCCGTGGATAATGG & & \\
\hline \multirow{2}{*}{ mrkA } & $\mathrm{F}$ & AGTGCTTTCACCCCCTCCT & \multirow{2}{*}{862} & \multirow{2}{*}{$\begin{array}{l}\text { M55912.1 } \\
\text { This study }\end{array}$} \\
\hline & $\mathrm{R}$ & GAGTGACTGGGGTGAGCAAA & & \\
\hline \multirow{2}{*}{ iss } & $\mathrm{F}$ & TGTCACATAGGATTCTGCCGTT & \multirow{2}{*}{450} & \multirow{2}{*}{$\begin{array}{l}\text { NZ_RZLR01001650.1 } \\
\text { This study }\end{array}$} \\
\hline & $\mathrm{R}$ & TTCACCCTCAGAGAGAGGCT & & \\
\hline \multirow{2}{*}{ htrA } & $\mathrm{F}$ & CGTTCTGCCAGGATGGTTCT & \multirow{2}{*}{1071} & \multirow{2}{*}{$\begin{array}{l}\text { AJ430233.1 } \\
\text { This study }\end{array}$} \\
\hline & $\mathrm{R}$ & CCCCAATGATGACATCGCCT & & \\
\hline
\end{tabular}

\section{Results and discussion}

The distribution of bacterial genes according to the site of infection

The study showed that $K$. pneumoniae isolates were distributed regarding different site of collection as the following: $14(42.4 \%)$ isolate from wound, $15(45.4 \%)$ isolates from urine, $1(3.03 \%)$ isolate from blood,
$1(3.03 \%)$ isolate from ear swab,1(3.03) from stool and also $1(3.03 \%)$ isolate from vagina (Table 2,3$)$. The prevalence of, htrA, rmpA (42.4\%) (21) wound $m r k A$ and iss genes was (9\%), htrA (27\%) rmpA (21\%), mrkA $(21 \%)$, iss $(0 \%)$ urine. The total of gene htrA (78\%), $m r k A(21 \%), \operatorname{rmpA}(18 \%)$ and iss (12\%) (table 2).

The virulence-associated phenotypes including complement resistance, adhesion and invasion, and 
maintenance within macrophagesThese result was agreed with several previous studies like that established by Ravichitra et al. (2014) and Biradar et al. (2015), whose found that K. pneumoniae was the most typical member of Klebsiella spp. Which will cause severe infections.

Table 2: Distribution of Klebsiella pneumonia isolates according to the site of infection $(\mathrm{N}=33)$

\begin{tabular}{|l|l|l|}
\hline Site of infection & No. of K. pneumoniae & Percentage (\%) \\
\hline Wound & 14 & 42.4 \\
\hline Urine & 15 & 45.4 \\
\hline Blood & 1 & 3.03 \\
\hline Vagina & 1 & 3.03 \\
\hline Stool & 1 & 3.03 \\
\hline Ear swab & 1 & 3.03 \\
\hline Total & 33 & $100 \%$ \\
\hline
\end{tabular}

Table 3: The distribution of virulence factors genes among bacterial isolates according to site of infection.

\begin{tabular}{|l|l|l|l|l|}
\hline Source of isolate & htrA (\%) & rmpA (\%) & mrkA (\%) & iss (\%) \\
\hline Wound & $14 / 33(42.4 \%)$ & $7 / 33(21 \%)$ & $3 / 33(9 \%)$ & $3 / 33(9 \%)$ \\
\hline Urine & $9 / 33(27 \%)$ & $7 / 33(21 \%)$ & $1 / 33(3 \%)$ & $0 / 33(0 \%)$ \\
\hline Blood & $1 / 33(3 \%)$ & $1 / 33(3 \%)$ & $1 / 33(3 \%)$ & $1 / 33(3 \%)$ \\
\hline Vagina & $0 / 33(0 \%)$ & $1 / 33(3 \%)$ & $1 / 33(3 \%)$ & $0 / 33(0 \%)$ \\
\hline Stool & $1 / 33(3 \%)$ & $0 / 33(0 \%)$ & $0 / 33(0 \%)$ & $0 / 33(0 \%)$ \\
\hline Ear swab & $1 / 33(3 \%)$ & $1 / 33(3 \%)$ & $0 / 33(0 \%)$ & $0 / 33(0 \%)$ \\
\hline Total & $26(78 \%)$ & $17(51 \%)$ & $6(18 \%)$ & $4(12 \%)$ \\
\hline
\end{tabular}

Phenotypic detection of Virulence factors:

Virulence factors of Klebsiella pneumoniae demonstrated in this work included production of capsule, serum resistance and biofilm formation.

Capsule production:

The results all Klebsiella pneumoniae isolates(100\%) were positive for capsule production
These results in the present study agreed with power et al.,(2004), who found that capsule production is considered as an important virulence factors in bacterial pathogenicity because capsular material forms thick bundles of fibrillous structures covering the bacterial surface in massive layers.

\section{Biofilm Formation ability by Klebsiella} pneumoniae: 
Biofilm formation ability of $K$. pneumoniae was investigated in this study and the results showed that 2 isolates (10\%) strong, 9 isolates $(30 \%)$ had moderate capacity of biofilm formation, while only 15 isolates $(40 \%)$ were weak producers of biofilm, and non 7isolates $(20 \%)$.

Biofilm formation capacity of Klebsiella pneumoniae in the current study was tested on ELISA plate as an inanimate object. $K$. pneumoniae has a tendency to form biofilms on biotic and abiotic surfaces, including catheters and other medical devices, which is a contributing factor to their antibiotic resistance (Van Laar et al.,2014). Several factors required for biofilms formation have been identified in K. pneumoniae clinical isolates from the gastrointestinal tract and in strains that are associated with pneumonia and urinary tract infection (Wu et al.,2011).

The bacterial isolates were also tested for serum resistance at an absorbance of $630 \mathrm{~nm}$. The results showed that $15 \%$ of Klebsiella pneumonia,

Molecular characterization of $K$. pneumoniae

\section{isolates:}

\section{Detection of $r m p A$, iss and $m r k A, h t r A$ genes:}

four selected genes were investigated in this study to explore their role in the pathogenesis of Klebsiella pneumoniae associated diseases. These genes were iss gene (4) positive, $m r k A$ gene (5) positive, and $h \operatorname{tr} A$ gene (26) positive, $r m p A$ gene (17) by PCR technique.

In this study mrkA (Mannose resistant Klebsiella polypeptide A) gene 862BP five represent positive isolates disagree with ( Nathalie et al;2003) Twentythree (69.7\%) of the 33 type strains tested type 3 fimbriae may provide different functions for the bacteria, one enabling a specific receptor-ligand interaction with host cells and tissues and the other facilitating attachment to abiotic surfaces and then outgrowth of the bacteria as an efficient biofilm in several environments. Type 3 fimbriae-dependent adherence may be the first step in $K$. pneumoniae colonization of non-biological surfaces and biofilm development and thus could be a major virulence determinant in nosocomial infections. However, since some highly adherent strains (Nathalie et al;2003)

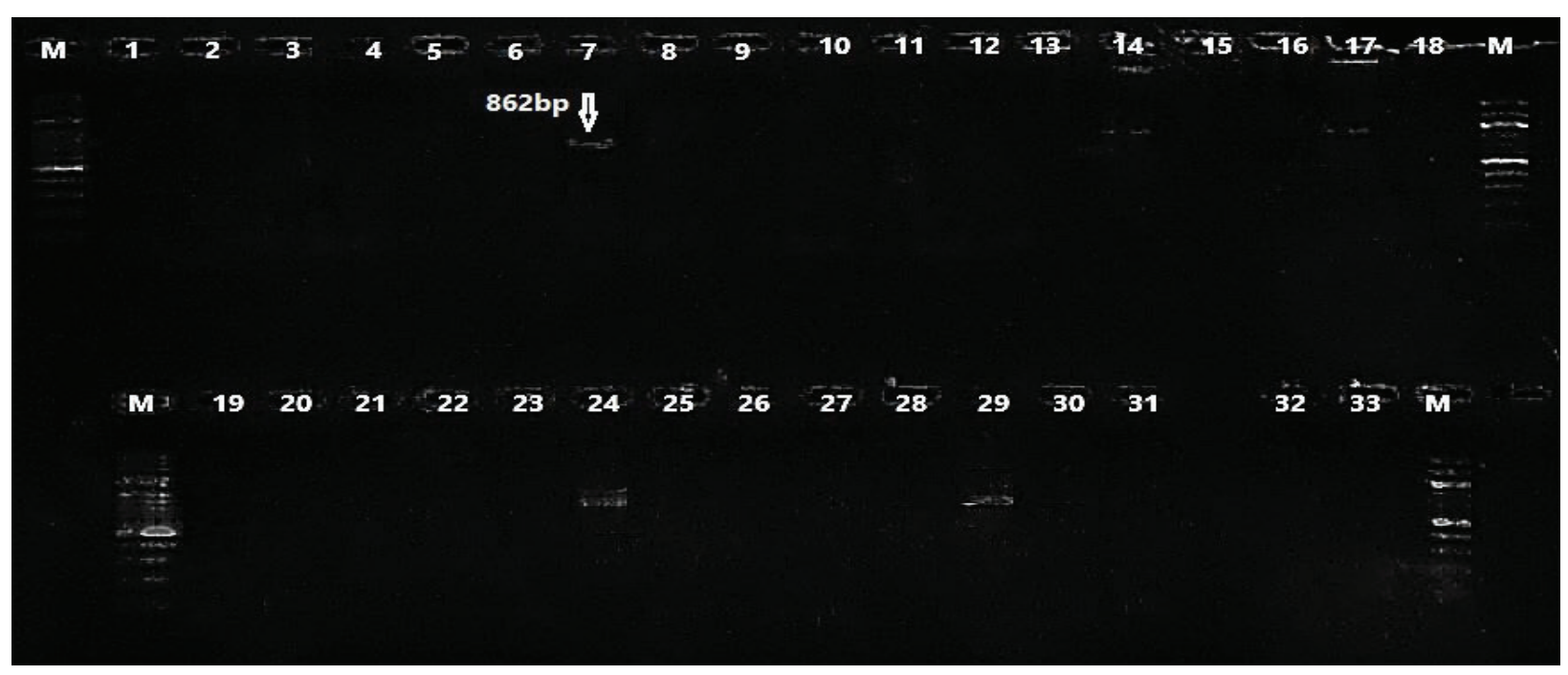

Figure 1: performed of polymerase chain reaction with mrkA gene of $K$. pneumoniae isolates, 1.5 agarose. $72 \mathrm{volts} 60$ min: M:100bp DNA marker Lane $(7,14,17,24,29)$ Amplified PCR product of $m r k A$ gene.

The presence of rmpA gene has been associated with the hypermuscoviscosity phenotype in K. pneumoniae, and was found to be more prevalent in liver abscess strains than in bacteremia isolates (Yu et al., 2006).

In this study mrkA (Mannose resistant Klebsiella polypeptide A) gene 862BP five represent positive isolates disagree with ( Nathalie et al;2003)Twentythree (69.7\%) of the 33 type strains tested type 3 fimbriae may provide different functions for the bacteria, one enabling a specific receptor-ligand interaction with host 
cells and tissues and the other facilitating attachment to abiotic surfaces and then outgrowth of the bacteria as an efficient biofilm in several environments. Type 3 fimbriae-dependent adherence may be the first step in $K$. pneumoniae colonization of non-biological surfaces and biofilm development and thus could be a major virulence determinant in nosocomial infections. However, since some highly adherent strains (Nathalie et al;2003)

It was first reported in 1989 by (Nassif et al., 1989) and is the first one explained that remove of the rmpA gene can decrease about 1000 fold of virulence in mouse lethality tests, in spite there was a positive relationship between virulent $K$. pneumoniae and the presence of rmpA gene, but this gene remained unknown for many years.

The rmpA gene is the most frequently occurring ones, the latter being associated with $K$. pneumoniae hypermucoviscosity and high virulence (Chang et al.,2013). The rmpA gene is located on a 180-kilo base of multi copy plasmid, is a strong virulence plasmid and it has an important role in expressing the mucoid phenotype of $K$. pneumoniae (Suescun et al., 2006).

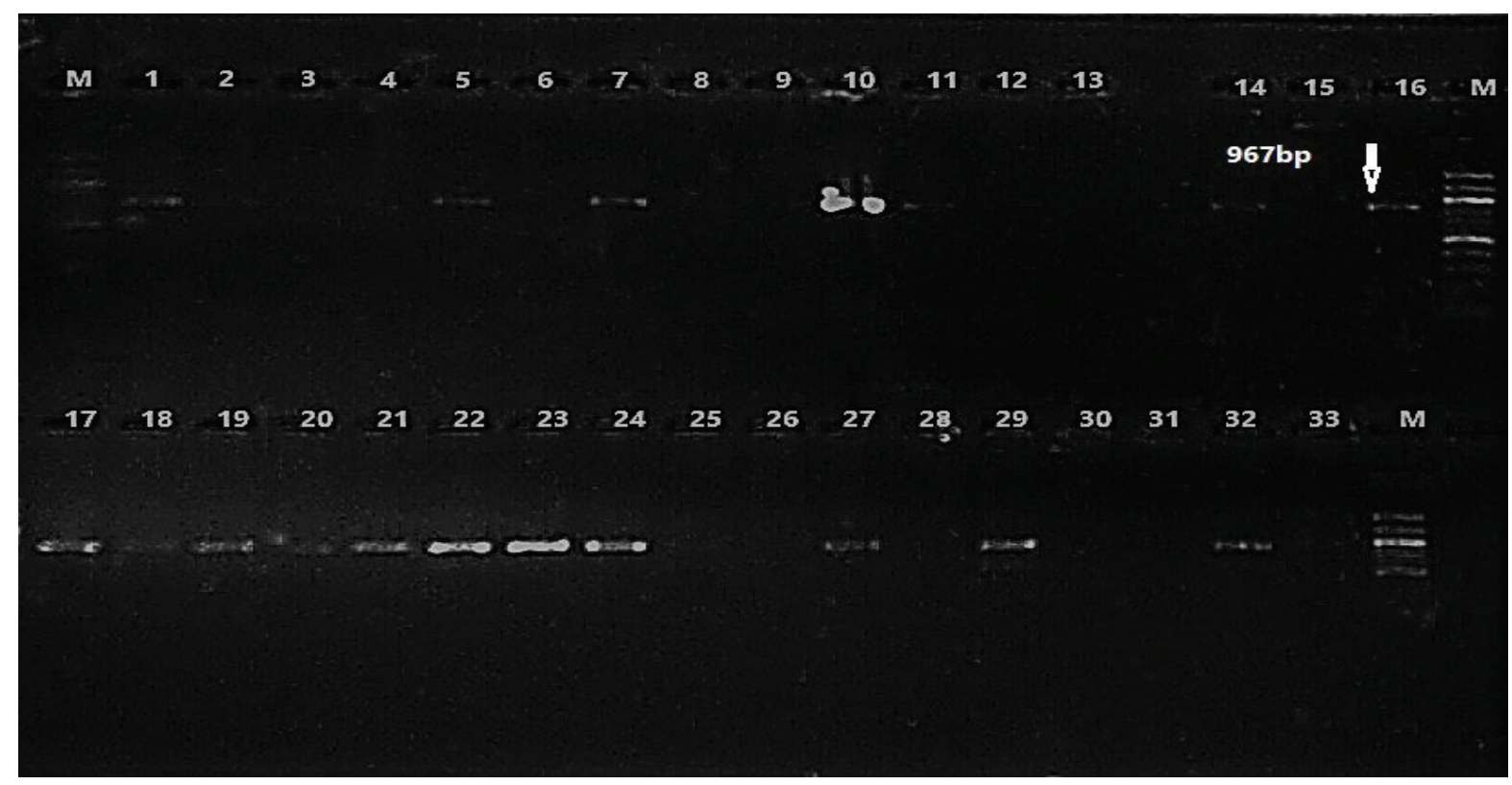

Figure 2: performed of polymerase chain reaction with rmpA gene of K. pneumoniae isolates, 1.5 agarose. 72 volts 60 min: M:100 bp DNA marker Lane $(1,5,7,10,14,16,17,18,19,20,21,22,23,24,27,29,32)$ Amplified PCR product of rmpA gene.

HtrA: htrA (high temperature requirement A) gene is now of interest because htrA mutant strains of several gram-negative bacteria have been shown to be attenuated in animal models and can also be used as vaccines ${ }^{7}$. In this study htrA gene1071BP (26) represent positive isolates.
Downregulation of bacterial transmigration by $H$. pylori htrA mutations. Polarized monolayers of MKN28 cells were infected by $H$. pylori wild-type or mutant strains. The mutant strains (N6 $\Delta h t r \mathrm{~A}, \mathrm{~N} 6 \Delta h t r \mathrm{~A} /$ $h t r \mathrm{AN} 6$ and N6 $\Delta h t r \mathrm{~A} / h t r \mathrm{AS} 221 \mathrm{AN} 6$ encode the same SecAR837K mutant variant). Transmigrated bacteria were quantified by counting CFUs. All experiments were done in triplicates (Anna Zawilak et al.,2019). 


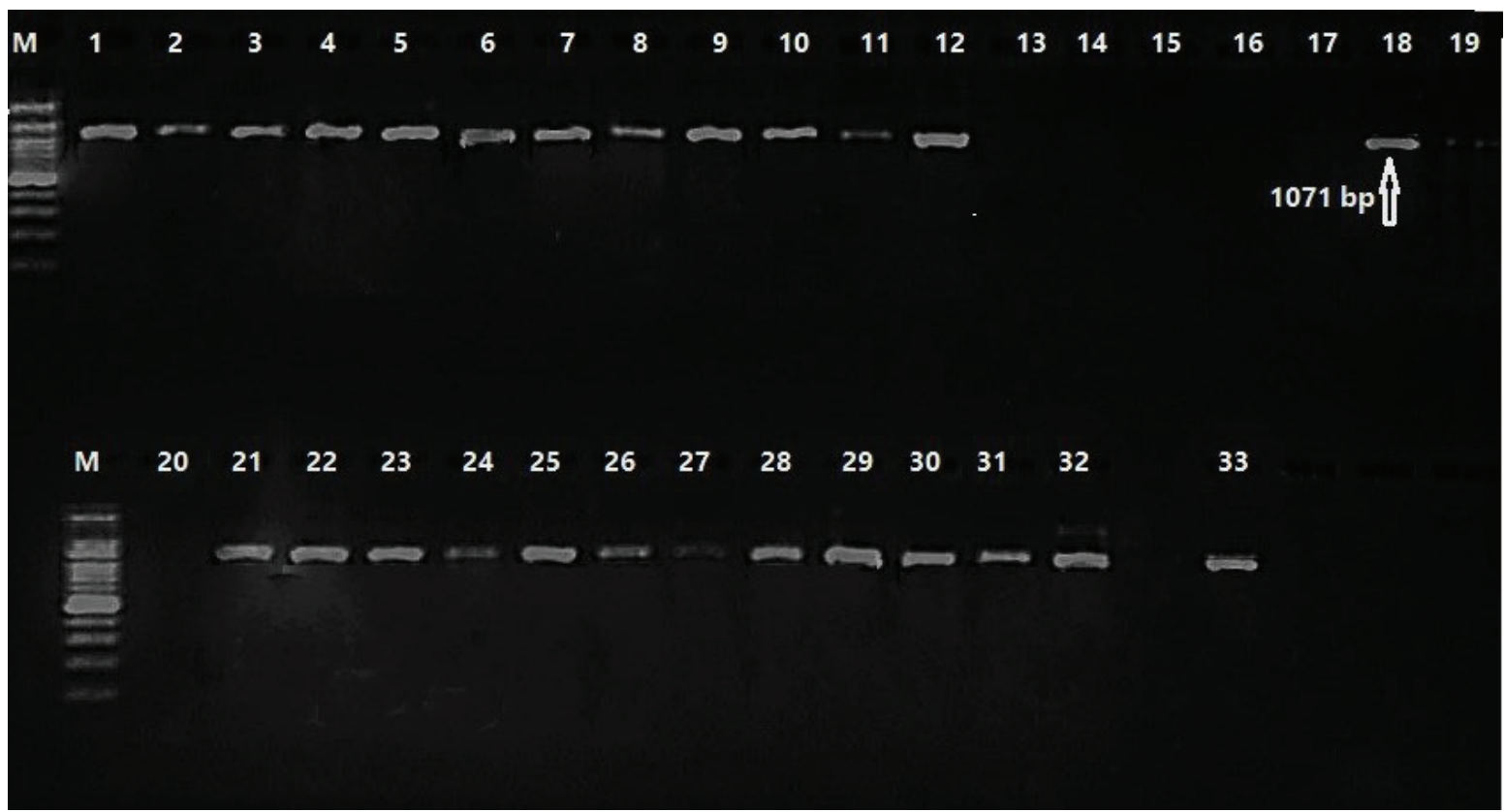

Figure 3: performed of polymerase chain reaction with hrtA gene of $K$. pneumoniae isolates, 1.5 agarose. 72 volts 60 min: M: 100 bp DNA marker Lane $(1,2,3,4,5,6,7,8,9,10,11,12,18,21,22,23,24,25,26,27,28,29,30,31,32,33)$ Amplified PCR product of $h t r A$ gene.

The increased serum survival protein (iss gene) has an action in defense to serum complement Nolan, (2003). The iss has been known for its activity in virulence of extraintestinal pathogenic Escherichia coli strains (ExPEC) Hassan, (2011). The iss gene is recognized as one of the most common virulence genes in extraintestinal pathogenic strains in poultry. The occurrence of immune resistance among Enterobacteriaceae family was a cause to study the prevalence of genes associated with serum resistance among Enterobacteriaceae

Among all patients diagnosed with $K$. pneumoniae, Lin et al., (2015). The prevalence of, $h$ trA, rmpA (42.4\%) (21) wound $m r k A$ and iss genes were (9\%), htrA (27\%) rmpA (21\%), mrkA (21\%), iss (0\%) urine. the total of gene htrA (78\%), mrkA (21\%), rmpA (18\%) and iss $(12 \%)$.

The virulence-associated phenotypes including complement resistance, adhesion and invasion, and maintenance within macrophages These results was agreed with several previous studies like that established by Ravichitra et al. (2014) and Biradar et al. (2015), whose found that $\mathrm{K}$. pneumoniae was the most typical member of Klebsiella spp. Which will cause severe infections.
Financial Disclosure: There is no financial disclosure.

\section{Conflict of Interest: None to declare.}

Ethical Clearance: All experimental protocols were approved under the Department of Biotechnology and all experiments were carried out in accordance with approved guidelines.

\section{References}

1. Hao C, Abhilasha K, Duy P, Christine J. A highresolution genomic analysis of multidrugresistant hospital outbreaks of Klebsiella pneumoniae. You have full text access to this OnlineOpen article EMBO Molecular Medicine. 2015; 7: 3.

2. Aher T, Roy A, Kumar P. Molecular detection of virulence genes associated with pathogenicity of Klebsiella spp. isolated from the respiratory tract of apparently healthy as well as sick goats. J. Veterinary Med. 2012; 67(4): 249-252.

3. Kim H, Chon J, Kim Y. Prevalence and characterization of extended-spectrum- $\beta$ lactamase-producing Escherichia coli, and Klebsiella pneumoniae, in ready-to-eat vegetables. Int. J. Food Microbiol. 2015; 207: 83-86.

4. Dubey D, Raza F, Sawhney A, Pandey A. Klebsiella pneumoniae renal abscess syndrome: a rare case 
with metastatic involvement of lungs, eye, and brain. Case Reports in Infect. Dis. 2013; 1-3.

5. Ravichitra KN, P Hema, S Subbarayudu. Isolation and antibiotic sensitivity of Klebsiella pneumoniae from pus, sputum and urine samples. 2014;3(3) :115-119.

6. Murphy CN, Clegg S. Klebsiella pneumoniae and type 3 fimbriae: nosocomial infection, regulation and biofilm formation. Future Microbiol. 2012; 7(8): 991-1002.

7. Chatfield S, H Strahan, D Pickard, IG Charles. Molecular characterization of the PhoPQPmrDPmrAB mediated pathway regulating polymyxin "B" resistance in Klebsiella pneumoniae CG43. J. Biomedical Sci. 2010; 17(60): 2-6.

8. Biradar S, C Roopa. Isolation and Antibiogram of Klebsiella species from Various Clinical Specimens. 2015; 4(9): 991-995.

9. Chiu S, Wu T, Chuang $\mathrm{Y}$, Lin J. National surveillance study on carbapenem Non-susceptible Klebsiella pneumoniae in taiwan: the emergence and rapid dissemination of $\mathrm{kpc}-2$ carbapenems. Plos One. 2013; 8(7): 1-7.

10. Cortes G, Borrell N, de Astorza B. Molecular analysis of the contribution of the capsular polysaccharide and the lipopolysaccharide $\mathrm{O}$ side chain to the virulence of Klebsiella pneumoniae in a murine model of pneumonia. J. Infect. Immun. 2002; 70(5): 2583-2590.

11. Guo Y, Wang S, Zhan L. Microbiological and clinical characteristics of hypermucoviscous Klebsiella pneumoniae isolates associated with invasive infections in China. Front. Cell. Infect. Microbiol. 2017; 7: 24.

12. Kadouri D, Venzon N, O'Toole O. Vulnerability of Pathogenic Biofilms to Micavibrio aeruginosavorus. Applied and Environmental Microbiology. 2007; 73: 605-14.

13. Kwon A, Park G, Ryu S. Higher biofilm formation in multidrug-resistant clinical isolates of Staphylococcus aureus. Int. J. Antimicrob. Agents. 2008; 32: 68-72.
14. Linares J, Lopez J, Camafeita E. Overexpression of the multidrug efflux pumps MexCD-OprJ and MexEF-OprN is associated with a reduction of type III secretion in Pseudomonas aeruginosa. J Bacteriol. 2005;187: 1384-1391.

15. Power, M, J Littefield, DM Gorden. Phenotypic and genotypic characterization of Encapsulated Escherichia coli isolated from blooms in two Australian lakes. Env. Microbiol. 2004; 7(5): 631640.

16. Quinn J. Clinical problems posed by multi-resistant non-fermenting gram-negative pathogens. Clin Infect Dis. 1998; 27: 117-S124.

17. Soensen U. Pneumococcal polysaccharide antigens: capsules and C-polysaccharide. Danish. Med. Bull. $1995 ;$ 42:47-53.

18. Stepanovic S, Vukovic D, Hola V. Quantification of biofilm in microtiter plates: overview of testing conditions and practical recommendations for assessment of biofilm production by staphylococci. APMIS. 2007; 115: 891-899.

19. Struve C, Bojer M, Krogfelt KA. Identification of a conserved chromosomal region encoding Klebsiella pneumoniae type 1 and type 3 fimbriae and assessment of the role of fimbriae in pathogenicity. Infect. Immun. 2009; 77(11): 5016-5024.

20. Van Laar T, Chen T, Childers B. Genome sequence of a multidrug-resistant strain of Klebsiella pneumoniae, BAMC 07-18, isolated from a combat injury wound. Genome Announc. 2014; 2(6): 1223 1230.

21. Vemula S, Vadde R. Prevalence of ESBL-producing Klebsiella pneumoniae isolates in Tertiary Care Hospital.ISRNMicrobiology. 2011.

22. Vuotto C, Francesca L, Maria P, Gianfranco D. Antibiotic Resistance Related to Biofilm Formation in Klebsiella pneumoniae. Pathogens. 2014; 3: 743758.

23. Wu M, Lin T, Hsieh P. Isolation of genes involved in biofilm formation of a Klebsiella pneumoniae strain causing pyogenic liver abscess. PLoS One. 2011; 6(8): e23500. 